\title{
Familial predisposition to recurrent mutations causing Huntington's disease: genetic risk to sibs of sporadic cases
}

\author{
Y P Goldberg, S E Andrew, J Theilmann, B Kremer, F Squitieri, H Telenius, \\ J D Brown, M R Hayden
}

\begin{abstract}
Huntington's disease (HD) is associated with expansion of a CAG repeat in a new gene. We have recently defined a premutation in a paternal allele of 30 to 38 CAG repeats in the HD gene which is greater than that seen in the general population ( $<30$ repeats) but below the range seen in patients with $\mathrm{HD}(>38)$. These intermediate alleles are unstable during transmission through the germline and in sporadic cases expand to the full mutation associated with the clinical phenotype of HD. Here we have analysed three new mutation families where, in each, the proband and at least one sib have CAG sizes in the HD range. In one of these families, two sibs with expanded CAG repeats are both clinically affected with HD, thus presenting a pseudorecessive pattern of inheritance. In all three families the parental intermediate allele has expanded in more than one offspring, thus showing a previously unrecognised risk of inheriting HD to sibs of sporadic cases of HD.

( $\mathcal{F}$ Med Genet 1993;30:987-90)
\end{abstract}

George Huntington, in his classic description in the Medical and Surgical Reporter in 1872, noted that Huntington's disease seems to obey 'fixed laws' when he observed that in all instances "this disease appears to be transmitted from parent to child". 'It is not surprising that George Huntington did not describe any new mutations causing $\mathrm{HD}$ as these have been thought to be extremely rare, with the mutation rate in HD among the lowest known for any genetic disorder. ${ }^{2-4}$ In part this has reflected the fact that to diagnose a new mutation for HD very strict criteria have been developed including the fact that the parent must live beyond the expected age of onset and that paternity should be confirmed. ${ }^{5}$ Another factor that previously excluded the identification of a new mutation causing $\mathrm{HD}$ has been a family history of $\mathrm{HD}$ with the presence of $\mathrm{HD}$ in a sib of the proband. In these instances, even in the absence of HD in the parents, this has been taken as presumptive proof either that the parents are not as stated, or that one of the parents has not lived long enough to manifest signs and symptoms of this illness.

In our recent study of 21 new mutations causing $\mathrm{HD},{ }^{6}$ we have shown that $\mathrm{CAG}$ repeat expansion underlies the vast majority of cases with sporadic HD, similar to that seen in persons with a family history of this disorder. We have shown the existence of an intermediate allele of 30 to $38 \mathrm{CAG}$ repeats in the fathers of sporadic cases of $\mathrm{HD}$. These intermediate alleles are unstable during transmission through the germline and in the sporadic cases expand to the full mutation associated with the clinical phenotype for HD.

Here we provide a more detailed analysis and clinical histories on three of these families. We show that expansion of the premutation to the full mutation associated with HD also occurs in some sibs of the probands with sporadic HD. This constitutes, therefore, a previously unrecognised genetic risk of inheriting HD to sibs of sporadic cases of HD.

\section{Methods}

PATIENT SELECTION

Probands with clearly documented HD were selected from our database by virtue of not having a family history of HD in the parents and where the parents had lived beyond the expected age of onset of HD (usually greater than 60 years without any manifestations of the disease) and where paternity of the sporadic case had been confirmed. Further evaluation of the family was obtained through detailed personal or telephone interviews and through clinical examination.

\section{PCR ANALYSIS}

Genomic DNA was isolated from leucocytes by standard extraction procedures. ${ }^{7}$ PCR amplification of the HD CAG repeat was performed as described previously ${ }^{8}$ using primers HD344 ( $5^{\prime}$ CCTTCGAGTCCCTCAAGTCCTTC $3^{\prime}$ ) and HD482 (5' GGCTGAGGAAGCTGAGGAG $3^{\prime}$ ). PCR conditions were $2 \mathrm{mmol} / 1 \quad \mathrm{MgCl}_{2}, \quad 50 \mathrm{mmol} / 1 \mathrm{KCl}$, $20 \mathrm{mmol} / 1$ Tris, $\mathrm{pH} 8.4,3.5 \%$ formamide, $15 \%$ glycerol, $200 \mu \mathrm{mol} / 1$ of each dNTP, $10 \mathrm{pmol}$ of each primer, and $1.25 \mathrm{U}$ of $\mathrm{Taq}$ DNA polymerase per $25 \mu \mathrm{l}$ reaction. Thermal cycling conditions were $95^{\circ} \mathrm{C}$ for three minutes followed by 30 cycles of $94^{\circ} \mathrm{C}$ for one minute, $62^{\circ} \mathrm{C}$ for one minute, $72^{\circ} \mathrm{C}$ for one minute, with a final extension at $72^{\circ} \mathrm{C}$ for seven minutes. PCR products were resolved as previously described on $6 \%$ polyacrylamide gels. ${ }^{9}$

\section{Results}

We recently reported on 21 new mutations for $\mathrm{HD}$ where the parents lived beyond the 
expected age at onset in the family and did not show any signs or symptoms of HD. We have identified three out of the 21 families where, in each, the father had an allele size of between 35 and 38 CAG repeats which on more than one occasion has expanded during transmission through the germline with the result that more than one offspring has a repeat length in the range seen in affected persons with $\mathrm{HD}$ (figure A-C).

The structure of these families has been changed to protect the confidentiality of family members.

\section{FAMILY 1}

The proband presented in her early twenties with mild speech disturbance and personality change. Associated with this, a mild involuntary movement disorder was evident, particularly affecting the hands and legs. With time, the disease showed inexorable progression with increasing chorea and further disturbance in gait. Associated with this were significant

A

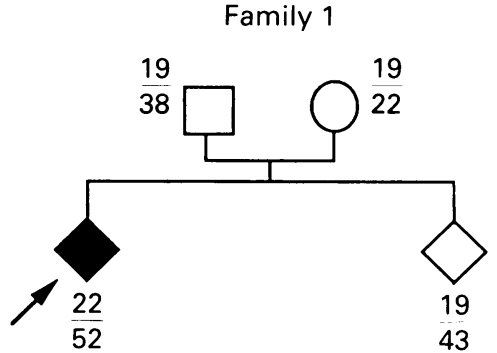

B

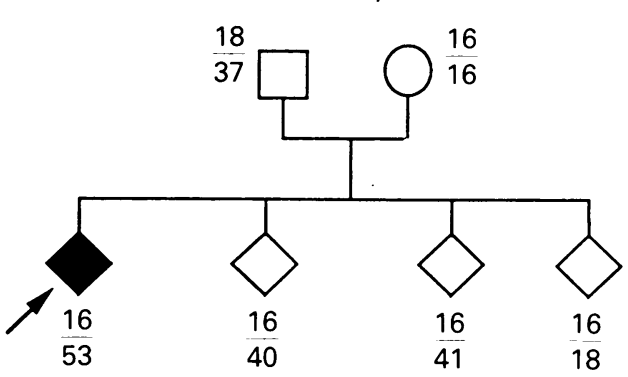

C

Family 3

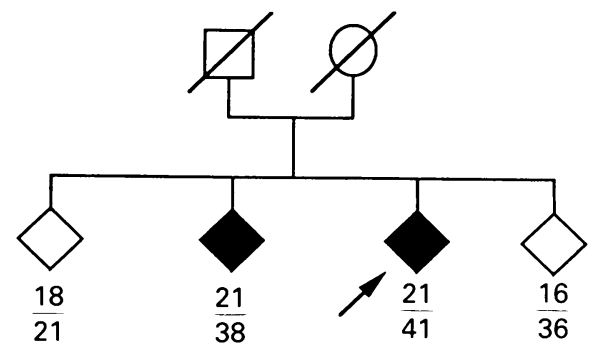

Pedigrees showing trinucleotide repeat length in new mutation families. $C A G$ repeat sizes of lower and upper alleles are shown. ( $A$ ) Family 1. The intermediate allele of $38 C A G$ repeats in the father has expanded to 52 in the proband and 43 in the sib. (B) Family 2. The intermediate allele of 37 repeats in the father has expanded to 53 repeats in the proband and to 40 and 41 in two other sibs. (C) Family 3. Two sibs have CAG repeat sizes in the $H D$ range and have clinical features of $H D$, mimicking a recessive pattern of inheritance. personality changes. By her early thirties this patient showed a more rigid phenotype and became anarthric. She had classical clinical signs consistent with the natural history of HD.

CT examination showed an enlargement of the lateral ventricles and a disturbance of the $\mathrm{FH} / \mathrm{CC}$ ratio consistent with caudate nucleus atrophy. Examination with positron emission tomography (PET) showed impaired uptake of glucose in the basal ganglia consistent with this diagnosis. Examination of her peripheral smear showed no acanthocytosis and serum copper and caeruloplasmin were normal.

Detailed family history showed no evidence for HD in either of her parents. Both parents lived beyond the age of 60 and had no manifestations of this illness. Furthermore, detailed family history on both sides of the family over five generations yielded no evidence for any progressive neurological disorder in any family relative. Paternity studies with HLA typing confirmed that this offspring was the child of her supposed parents.

DNA analysis showed that the proband had alleles with CAG repeat lengths of 52 and 22 for the upper and lower alleles respectively (figure A). The father of the proband died in his mid sixties. Detailed neuropathological examination in the father provided no evidence for HD; however, repeat analysis on DNA extracted from brain showed one CAG allele in the normal range (19 repeats) and one of intermediate size (38 repeats). A sib of the proband was found to have CAG repeat lengths of 43 and 19 for the upper and lower alleles respectively. This sib is completely asymptomatic at present and shows no signs of this disorder. Thus, in this family the intermediate allele of 38 in the father had expanded to 52 in one of the offspring and 43 in another.

FAMILY 2

This patient (figure B) first presented in the late twenties with significant depression and mild involuntary movements. Clinical examination showed evidence of chorea, impaired eye movements, hyperreflexia, and a gait disturbance. The disease showed a significant progression over the next 10 years with increasing chorea, progressive intellectual decline, and further disturbances in gait, speech, and swallowing. CT scan showed caudate nucleus atrophy. This patient died approximately 14 years from the time of onset and a detailed pathological examination found neuronal loss in the caudate nucleus associated with astrocytosis, thus confirming the diagnosis of HD.

Detailed interview and examination of the parents showed that both parents were over 70 years old without signs or symptoms of any neurological illness. There was no evidence for a progressive neurological disorder in either parent or any of their ancestors.

The proband is one of a sibship of four where no other sibs manifest any signs or symptoms of this illness. Detailed paternity studies using polymorphic DNA markers confirmed that all the children were the offspring 
of these parents. The father and mother had upper alleles of 37 and 16 respectively, while the proband had an upper allele size of 53 repeats. Molecular analysis of the three sibs showed that one sib had allele sizes within the normal range at 16 and 18 respectively, while two other sibs had upper alleles consistent with the affected range for $\mathrm{HD}$, of 40 and 41 respectively (figure $B$ ). Detailed haplotype analysis using markers at D4S127, D4S95, and an $A c c$ I polymorphism within the gene (manuscript in preparation) showed that the upper allele in the father had expanded to the allele size of 53 in the proband and to 40 and 41 in two of the other offspring.

\section{FAMILY 3}

The proband in family 3 (figure C) had onset of clinical features with clumsiness, involuntary movement disturbances, and personality change aged 39 . The illness followed a progressive course and the diagnosis of possible HD was made based on the clinical presentation and evidence for caudate nucleus atrophy on CT scan. The parents were both dead but neither of them had suffered from any neurological disorder. Both had lived beyond the age of 50 . Both sets of grandparents had lived beyond the age of 60 and detailed history showed no evidence of any neurological disorder in any first, second, or third degree family relatives.

A sib of the proband first developed involuntary movements and personality changes aged 49. This was associated with intellectual decline and memory loss. The movement disorder was progressive, associated with the characteristic gait and this patient also had evidence for caudate nucleus atrophy on CT scan. The diagnosis of HD was made despite the fact that neither of the parents manifest this illness. The clinical phenotype was very similar to that seen in the proband although this occurred 10 years later.

Molecular analysis showed that all children in this sibship were offspring of the same parents. The CAG repeat length could be determined in all four sibs. The proband had an upper allele of 41 , while the affected sib had an upper allele of 38 repeats (figure C). Another sib had an upper allele size in the intermediate range (36 repeats), while another had both allele sizes within the normal range of 18 and 21.

\section{Discussion}

In the past a family history of HD in a sib, even in the absence of a history of HD in the parents, immediately excluded a new mutation causing this disorder in this family. In these three families we have shown that an intermediate CAG allele within the HD gene present in one of the parents has undergone expansion in at least two offspring, resulting in an allele size within the range seen in persons with HD. This expansion has occurred during transmission through the germline.

We have previously shown that in all seven families in whom the transmitting parent could be identified, the transmitting parent was a male. ${ }^{6}$ Furthermore, the parent with the intermediate allele was of an advanced age ${ }^{6}$ (mean 36.7 years, range 29-55) at the time of birth of the sporadic case of HD, which is similar to that seen in the parents of other patients representing new mutations for autosomal dominant disorders. ${ }^{11-13}$ Thus, several factors appear to be predisposing to recurrent CAG expansion in offspring into the range expected to result in clinical signs of HD. These include the presence of the intermediate allele or premutation in the range greater than that seen in the general population $(>30 \mathrm{re}-$ peats) but below the range seen in most patients with HD. Other factors include transmission through the male germline and advanced paternal age. In family 3 , the presence of $\mathrm{HD}$ in two sibs and no clinical features in either parent would lead to the appearance of pseudorecessive inheritance for HD which is, however, the result of recurrent expansion of the intermediate allele in different offspring. Clearly, it is apparent from these studies that there is a risk of inheriting an expanded allele for sibs of sporadic cases of HD.

The precise estimates of the likelihood of expansion of the intermediate allele during transmission through the germline is uncertain. In our previous report, ${ }^{6}$ we showed 10 expansions of 24 meioses ( $42 \%$ ) which would indicate that the maximum risk to a sib of a sporadic case of inheriting HD is likely to be not greater than $25 \%$. This would reflect the likelihood that they would inherit the chromosome which contains the intermediate allele in the parent and that this CAG has undergone expansion. Clearly, however, larger studies in additional families are needed to provide a precise estimate of risk for these sibs. The important point is the recognition that in families with a recurrence of HD in a sib without any manifestation in the parents, this may represent recurrent expansion of an intermediate allele from an unaffected parent. The failure of inclusion of such cases in estimates of new mutation rate would also significantly lower the calculation of mutation rates for this disorder which is likely therefore to be higher than previously estimated.

This study is biased towards identification of families in whom there is a predisposition to expansion of the intermediate allele from parent to child since we have selected these families through an affected proband. The frequency of the intermediate allele in the general population is unknown. Only a proportion of persons with the intermediate allele, however, are likely to manifest CAG expansion during transmission through the germline. Once the factors contributing to CAG expansion are understood, this might provide further information for persons with intermediate alleles in the families in whom sporadic cases have previously been identified. In particular if it is confirmed that advanced paternal age is one factor contributing to expansion of this intermediate allele, this would be important information for such family members so that this 
could be incorporated into their plans for procreation.

It is noteworthy that in the three families described, the intermediate allele is very close to the range seen in some affected persons, usually of late onset. This might represent evidence that the size of the intermediate allele is a factor in determining its propensity to expansion. At the time of assessment, the parents with the intermediate alleles were not showing any signs or symptoms of HD. However, we cannot exclude the possibility that they might manifest some signs at a very advanced age.

This study shows that on rare occasions where there is no history of HD in the parents, the illness could manifest in more than one offspring giving the appearance of pseudorecessive inheritance. In this instance, the explanation might not reflect a non-paternity event but, as shown here, more likely represents expansion of an intermediate allele in recurrent pregnancies. The investigation of CAG repeat length in families with unusual family histories has provided new insights into the patterns of expansion of this repeat and has provided a biological basis for the unusual family histories for HD seen in some families.

We are grateful for the clinical details and DNA samples provided by members of the Canadian HD Collaborative Group on predic- tive testing. This work is supported by the MRC (Canada), the Canadian Genetic Disease Network, and the Huntington Society of Canada. YPG is a Sandoz postdoctoral fellow. $\mathrm{BK}$ and $\mathrm{HT}$ are MRC postdoctoral fellows. FS is supported by the Instituto Scientific Sanatrix. MRH is an established investigator for the BC Children's Hospital.

1 Huntington G. On chorea. Med Surg Rep 1872;26:317-21.

2 Harper PS. Huntington's disease. London: Saunders, 1991.

3 Hayden MR. Huntington's chorea. New York: Springer-

4 Vogel F, Motulsky A. Human genetics. 2nd ed. New York: Springer-Verlag, 1986.

5 Stevens D, Parsonage M. Mutation in Huntington's chorea. f Neurol Neurosurg Psychiatry 1969;32:140-3.
Stevens D, Parsonage $M$. Mutation in Huntingto

6 Goldberg YP, Kremer B, Andrew SE, et al. Molecular analysis of new mutations for Huntington disease: intermediate alleles and sex of origin effects. Nature Gene 1993;5:174-9.

7 Kunkel LM, Smith KD, Boyer SH, et al. Analysis of human $\mathrm{Y}$ chromosome specific reiterated DNA in chromosome variants. Proc Natl Acad Sci USA 1977 74:1245-9.

8 Andrew SE, Goldberg YP, Kremer B, et al. The relationship between trinucleotide (CAG) repeat length and clini1993;4:398-403.

9 Goldberg YP, Andrew SE, Clarke LA, Hayden MR. A PCR method for accurate assessment of trinucleotide PCR method for accurate assessment of trinucleotide repeat expansion

10 Hayden MR, Martin WRW, Stoessl AJ, Clark C. Positron emission tomography in the early diagnosis of Huntington disease. Neurology 1986;36:888-94

11 Penrose LS. Parental age and mutation. Lancet 1955;ii:312. 2 Penrose LS. Parental age in achondroplasia and mongolism Am F Hum Genet 1957;9:167-9.

13 Murdoch J, Walker BA, McKusick VA. Parental age effects on the occurrence of new mutations for the Marfan syndrome. Ann Hum Genet 1972;35:331-6. 